\title{
Rapidly progressive stage IVB mycosis fungoides treated with low-dose total skin electron beam therapy
}

\author{
Mudit Chowdhary ${ }^{1,2}$ \\ Ahmad A Kabbani' \\ Parin Rimtepathip' \\ David A Cole Jr ${ }^{2}$ \\ David J Cohen'
}

'Division of Dermatology, Department of Internal Medicine, ${ }^{2}$ Division of Radiation Oncology, Department of Radiology, Mercer University School of Medicine, Macon, GA, USA
This article was published in the following Dove Press journal:

OncoTargets and Therapy

I July 2015

Number of times this article has been viewed
Correspondence: Mudit Chowdhary Division of Dermatology, Department of Internal Medicine, Mercer University School of Medicine, 308 Hospital Drive, Suite 200, Macon, GA 31217, USA Email mu.chowdhary@gmail.com
Abstract: Mycosis fungoides (MF) is the most common subtype of primary cutaneous T-cell lymphoma. Normally, MF has an indolent course although patients can progress to an advanced disease state (stages IIB-IVB). Advanced-stage disease is typically aggressive, leaving patients with debilitating symptoms and a decreased quality of life. Moreover, advanced-stage MF often proves refractory to therapy and carries a very poor prognosis. Total skin electron beam (TSEB) therapy is a well-established and successful treatment for early stage MF; however, its efficacy dramatically decreases with advanced-stage disease. In fact, TSEB in advancedstage MF is generally considered to be palliative. Current consensus guidelines recommend a dose of 30-36 Gy to be delivered in 8-10 weeks; however, limited studies exist to determine the ideal treatment in Stage IV MF. Herein, we describe a case of a 50-year-old male who developed rapidly progressive stage IVB (T3N3M1B0) MF and was treated with low-dose (24 Gy) TSEB over 8 weeks. The patient was not treated with any systemic therapy before starting TSEB due to the widespread nature and the speed of disease progression. Remarkably, our patient showed nearly complete (95\%) response of his MF with no apparent side effects from radiation. Furthermore, he has remained in remission over 4 years, requiring only a small boost to a few "shadowed" areas. Our case illustrates the benefit of using TSEB in stage IV MF. Additionally, our experience shows that low-dose TSEB can occasionally be efficacious in stage IV disease.

Keywords: mycosis fungoides, total skin electron beam therapy, low-dose, TSEB, advanced stage, stage IV, remission

\section{Introduction}

Mycosis fungoides (MF) is the most common primary cutaneous T-cell lymphoma (CTCL) with an incidence of 4.1 per 1,000,000 person-years in the USA. ${ }^{1} \mathrm{MF}$ is primarily an indolent and benign disease often confined to the skin for years on end. Patients classically present with persistent lesions that may range from localized scaly patches or widespread plaques (stages IA-II). Treatment response in these early stages is excellent and a majority of patients achieve complete remission with either skindirected or systemic therapies.

In some instances, MF may progress to a more advanced malignant disease (stages IIB-IVB). In those cases, patients develop skin tumors, generalized erythroderma, and nodal or visceral involvement. Overall prognosis in advanced disease is exceedingly poor with a median survival of only 2.47 years. $^{2}$ Additionally, many of these patients suffer from debilitating pruritus and a significantly decreased quality of life. ${ }^{3}$ Treatment options for advanced disease are often palliative, consisting of biologic 
response modifiers (retinoids, interferon [IFN]-alpha, and IFN-gamma), phototherapy, chemotherapy, and total skin electron beam (TSEB) radiotherapy with or without adjuvant therapy. ${ }^{4}$ Despite the number of potential treatment options, patients with advanced-stage MF typically prove refractory to therapy.

Herein, we describe a case of a 50-year-old male who developed rapidly progressive stage IVB (T3N3M1B0) disease and was successfully treated with low-dose TSEB radiotherapy.

\section{Case report}

A 50-year-old African-American male with no significant past medical history was referred to our clinic due to new onset diffuse, infiltrative hyperpigmented plaques covering his upper body (Figure 1A and D). The affected areas were edematous, pruritic, and oozing. Punch biopsy from these plaques suggested a benign inflammatory eczematous condition. The patient was therefore prescribed topical $0.1 \%$ triamcinolone acetonide for his acute flare.

The patient appeared to us again several months later with apparent disease progression. His eczematous lesions had now progressed to involve his entire body. Additionally, he had developed extensive tumor-like nodules throughout his body which were particularly prominent over the face and back (Figure 1B and E). The patient also reported a noticeable weight loss and difficulty in using his hands and feet due to the nodules.

Physical examination revealed extensive thickening of the skin among all regions of his body. The tumorous nodules appeared to be dry and cracking with excoriation. This time, punch biopsy revealed a dense nodular to diffuse lymphoid infiltrate with cytologic atypia. Shave biopsy showed nodular and diffuse proliferation of enlarged highly atypical lymphoid cells, frequent necrotic cells, and several mitotic figures in the superficial to midreticular dermis. Immunohistochemical
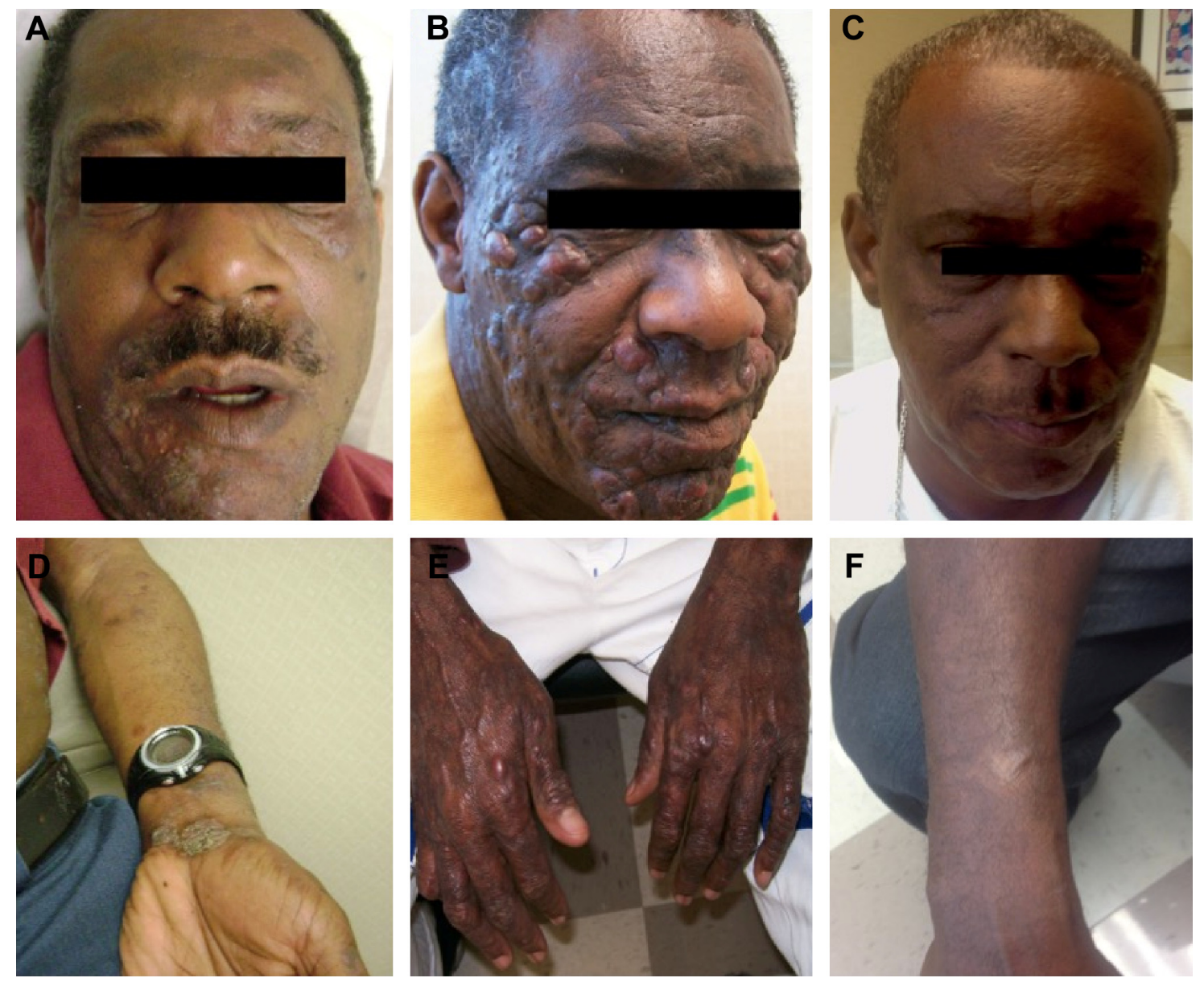

Figure I Disease course before and after TSEB.

Notes: (A, D) Initial presentation of patient revealed only eczematous changes. (B, E) Rapid disease progression within 3 months demonstrating multiple tumorous nodules throughout the body. (C, F) Complete disease response after 3 months following TSEB. (A-C) Progression of disease course in the face. (D-F) Progression of disease course in upper extremities.

Abbreviation: TSEB, total skin electron beam. 
staining of the skin displayed $24 \%$ atypical T-lymphocytes characterized as $\mathrm{CD}^{ \pm}, \mathrm{CD}^{ \pm}, \mathrm{CD}^{ \pm}, \mathrm{CD}^{ \pm}, \mathrm{CD}^{-}$, and CD45 $5^{ \pm}$. Subsequent gene rearrangement studies of the skin revealed distinct monoclonal peaks for the T-cell receptor gamma chain locus. These findings were consistent with a CTCL, specifically MF.

Axillary and inguinal lymph nodes were fixed and firm to palpation. The largest left axillary node was biopsied and analyzed. Biopsy revealed expansion of the paracortical area by a cellular infiltrate composed of abnormal lymphocytes with marked nuclear irregularities and brisk background mitotic activity. Flow cytometry revealed 13\% atypical T-lymphocytes. Malignant cells stained CD2-5 $5^{ \pm}, \mathrm{CD} 8^{-}$, CD25 $5^{ \pm}$, and CD45 ${ }^{ \pm}$. Peripheral blood was obtained, and it revealed no cytopenia. Specifically, the WBC count was $7.0 \mathrm{k} / \mu \mathrm{L}$, the $\mathrm{RBC}$ count was $5.05 \times 10^{12} / \mathrm{L}$, and the platelet count was $223 \mathrm{k} / \mathrm{mm}^{3}$. The differential revealed $70 \%$ neutrophils and 4.0\% lymphocytes (normal 19\%-48\%). Additionally, abnormal circulating lymphoid cells with cerebriform nuclear morphology (Sézary cells) were not present, indicating a lack of peripheral blood involvement.

In consideration of the nodal involvement, bone marrow aspirate was obtained and analyzed by flow cytometry. Bone marrow aspirate revealed 20.4\% neutrophils and 25\% lymphocytes. Flow cytometry was again positive (1\%) for atypical CD2- $5^{ \pm}, \mathrm{CD}^{-}$, and CD45 ${ }^{ \pm} \mathrm{T}$-lymphocytes, characteristics suspicious for bone marrow involvement. The patient was thus staged as stage IVB (T3N3M1B0) MF, based on criteria from the European Organization of Research and Treatment of Cancer and the International Society for Cutaneous Lymphoma. ${ }^{5}$

Due to the rapid progression and widespread nature of the patient's MF, clinical guidelines suggested ${ }^{6-8}$ the use of TSEB therapy. The patient underwent computed tomography of the chest, abdomen, and pelvis before beginning therapy. These were unremarkable asides from bilateral axillary and inguinal adenopathy. Initially, the patient was administered a trial of electron beam therapy to more symptomatic areas involving the skin over the back to which 18 Gy was delivered in ten fractions of $1.8 \mathrm{~Gy} /$ fraction. He tolerated this well with nice subjective and objective response, and we proceeded toward TSEB. The standard dose of 36 Gy over 10 weeks using the classic six-field technique ${ }^{9}$ was initially planned upon; however, the patient proved to be noncompliant and canceled his scheduled treatment plan after 8 weeks. Thus, only a total of 24 Gy was delivered to the total skin. Regardless, the patient responded very nicely with an impressive partial response $(>95 \%)$ of his lesions seen on initial follow-up from TSEB (Figure $1 \mathrm{C}$ and $\mathrm{F}$ ).
Furthermore, there was no expected skin darkening or systemic toxicity from the treatment. The patient required an additional boost dose of 12.5 Gy in five fractions of 2.5 Gy each to his right earlobe and buttocks 2 months following TSEB. These areas were missed from his initial therapy most likely as a result of difficulties from the classic TSEB technique. Since then, however, the patient has required no additional treatment and has shown a complete cutaneous response. ${ }^{10}$ Moreover, he has remained in remission from symptomatic cutaneous involvement over the past 4 years. The axillary lymphadenopathy has decreased on computed tomography scan, and the patient has not developed any new tumors on his body.

\section{Discussion}

MF is the most prevalent CTCL. The disease course for early stage disease is normally indolent, and many patients have mild disease or remain asymptomatic during their lifetime. Diagnosis can be challenging, as the early stages of the disease often resemble eczema, psoriasis, or dermatitis. The median duration from the onset of skin lesions to definitive diagnosis ranges from 8 to 10 years. Advanced-stage MF, however, carries an exceedingly poor prognosis. The median survival of patients with visceral involvement is only 2.5 years. $^{7}$

Skin-directed therapies are the first and mainstay treatment for stages IA-IIA MF. These consist of topical corticosteroids, psoralens and ultraviolet $\mathrm{A}$, topical retinoids, TSEB radiotherapy, and topical chemotherapy with agents such as carmustine and nitrogen mustard. These options are extremely successful for disease localized to the skin; however, multiple studies have reported conventional therapies to be refractory in advanced MF. ${ }^{11,12}$

The 2014 National Comprehensive Cancer Network Clinical Practice Guidelines in Oncology has defined firstline therapy for advanced-stage MF as local radiotherapy, TSEB radiotherapy, phototherapy, biologic response modifiers (IFN-alpha and IFN-gamma), and histone deacetylase inhibitors among others. ${ }^{13,14}$

TSEB therapy is a technically challenging treatment that requires special attention to avoid undue toxicity to healthy tissue. ${ }^{12}$ An electron beam is directed to penetrate the skin at a limited depth $(<2 \mathrm{~cm})$ to ensure minimal toxicity reaches visceral organs. MF cells are highly radiosensitive and can be effectively killed with low doses of radiation. ${ }^{7}$ Additionally, electron-beam irradiation is known to penetrate the skin more so than any other skin-directed treatments. ${ }^{15}$ Historically, TSEB of $\geq 30$ Gy has been highly effective and 
produced a better likelihood of a complete response. ${ }^{15}$ Thus, consensus guidelines have established a total treatment dose of 30-36 Gy, 4 or 5 days per week, over 8-10 weeks of TSEB in MF patients. ${ }^{16}$ Recently, a few studies have established equal clinical efficacy with lower-dose treatment protocols particularly in early stage MF. ${ }^{17,18}$ The first study, by Harrison et $\mathrm{al}^{17}$ demonstrated TSEB in doses of $10 \mathrm{~Gy}$ to $<20$ Gy and 20 Gy to $<30$ Gy were comparable to the standard dose regimen in terms of overall response and survival, although not in complete response or in response duration. Subsequently, Hoppe et $\mathrm{al}^{18}$ showed an impressive overall response rate of $88 \%$ and complete response rate of $27 \%$ with low-dose (12 Gy) TSEB over 3 weeks.

An added benefit to using TSEB is the general lack of heavy acute and late toxicity. Well-functioning patients are able to continue their prior normal activities of daily life. Although complete response is more frequently seen with the standard dose of TSEB, it is associated with greater toxicity. ${ }^{7}$ Most commonly skin lesions become acutely erythematous with dry desquamation. Patients can develop mild-to-moderate radiation dermatitis. Temporary scalp alopecia and nail stasis are also noted. Uncommon side effects can also include blisters on the fingers and feet, self-limiting anhidrosis, minor parotiditis, and gynecomastia in males. ${ }^{17}$ Unfortunately, many patients experience recurrent disease despite standard treatment. As such, the EORTC recommends that TSEB should not be administered more than three occasions in a patient's lifetime, restricting the use of this effective therapy. ${ }^{8}$ In comparison, lower-dose treatment may be advantageous by limiting dose-related toxicity and permitting the administration of multiple treatment courses. ${ }^{17,18}$

Although the efficacy of TSEB in the treatment of MF has been previously established, studies have rarely involved stage IV MF. ${ }^{2,17,18}$ In fact, stage IV disease treatment is normally considered to be palliative. However, this was in contrast to our case as we were able to achieve long-term remission in our patient. Initially, the standard dose of $36 \mathrm{~Gy}$ over a 10-week period was decided on; however, the patient proved to be noncompliant with his appointments. Thus, he ultimately received a lower dose (24 Gy) of radiation. Nevertheless, our patient remarkably showed almost complete regression of his lesions and tumors following TSEB. There has been no evidence of local or systemic radiation toxicity. Furthermore, he has remained in remission for the past 4 years requiring only a small radiation boost to his right earlobe and buttocks. It is common for some parts of the body to be "shadowed" and receive a lower total dose of from the classic TSEB technique. ${ }^{7}$
Our case shows how exquisitely radiosensitive MF truly is, as excellent clinical response was seen despite the widespread nature of this patient's disease. Certain factors potentially played a role in our success. Studies have indicated that significant blood involvement ( $>20 \%$ Sézary lymphocytes) is associated with an adverse prognosis; however, our patient displayed B0 blood involvement $(<5 \%$ Sézary cells). ${ }^{19}$ Alberti-Violetti et $\mathrm{al}^{2}$ found decreasing age ( $<65$ years) to be a negative risk factor in both overall survival and disease-specific survival in advanced-stage MF. Our patient was 50 years at the time of diagnosis and treatment. Another reason the treatment may have been so successful was the prompt administration of TSEB following diagnosis. Navi et $\mathrm{a}^{15}$ reported prior use of treatments reduced the possibility of response to TSEB. ${ }^{15}$ It is possible that native lymphoma cells respond better to TSEB. Additionally, patients in many studies have presented with symptoms for an extended period of time. Delay in treatment often leads to greater visceral involvement and poorer prognosis. However, due to the aggressiveness of our patient's disease, we directly proceeded to TSEB within 1 month of stage IVB (T3N1M1B0) diagnosis.

\section{Conclusion}

In conclusion, TSEB is a suitable and effective treatment option for patients with MF, with minimal systemic side effects. Despite its efficacy and relative safety, TSEB is infrequently used as a first-line treatment. This may be due to the technical difficulty of the procedure, the need for specialized therapeutic radiation centers, and even patient unwillingness to proceed with the treatment. Unfortunately, prognosis remains poor in advanced-stage MF and many patients experience disease relapse despite treatment. Regardless, we encourage clinicians and patients to utilize this therapy even in late-stage or rapidly progressing MF as numerous studies have shown the benefit of TSEB. Lowdose TSEB has also been shown to achieve good clinical response with a decreased risk of radiation toxicity, albeit in early stage disease. In this case, low-dose TSEB proved to be efficacious in achieving a complete remission. Nevertheless, further trials need to be established to determine whether low-dose TSEB is indeed beneficial for patients with stage IVB MF.

\section{Acknowledgment}

We would like to thank the staff of Dermatologic Surgery Specialists and Central Georgia Radiation Oncology for their assistance with this manuscript. 


\section{Disclosure}

Written consent from the patient was obtained before writing this manuscript. The authors report no conflicts of interest in this work.

\section{References}

1. Barton PT, Devesa SS, Anderson WF, Toro JR. Cutaneous lymphoma incidence in the United States: a population based study of 3,884 cases. Blood. 2009;113:5064-5073.

2. Alberti-Violetti S, Ralpur R, Schlichte M, Sui D, Duvic M. Advancedstage mycosis fungoides and Sezary syndrome: survival and response to treatment. Clin Lymphoma Myeloma Leuk. 2015;15(6):e105-e112.

3. Demierre MF, Gan S, Jones J, Miller DR. Significant impact of cutaneous T-cell lymphoma on patients' quality of life: results of a 2005 National Cutaneous Lymphoma Foundation Survey. Cancer. 2006; 107(10):2504.

4. Hinds GA, Alhariri J, Klein RQ, Wilson LD. Treatment of mycosis fungoides with total skin electron beam. Response and relapse by ethnicity and sex. Am J Clin Oncol. 2013;36(5):481-485.

5. Olsen E, Vonderheid E, Pimpinelli N, et al; ISCL/EORTC. Revisions to the staging and classification of mycosis fungoides and Sezary syndrome: a proposal of the International Society for Cutaneous Lymphoma (ISCL) and the cutaneous lymphoma task force of the European Organization of Research and Treatment of Cancer (EORTC). Blood. 2007; 110:1713-1722.

6. Jones GW, Kacinski BM, Wilson LD, et al. Total skin electron radiation in the management of mycosis fungoides: consensus of the European Organization for Research and Treatment of Cancer (EORTC) Cutaneous Lymphoma Project Group. J Am Acad Dermatol. 2002;47: 364-370.

7. Mazzeo E, Rubino L, Buglione M, et al. The current management of mycosis fungoides and Sezary syndrome and the role of radiotherapy: principles and indications. Rep Pract Oncol Radiother. 2014;19: 77-91.

8. Trautinger F, Knobler R, Willemze R, et al. EORTC consenus recommendations for the treatment of mycosis fungoides/Sezary syndrome. Eur J Cancer. 2006;42:1014-1030.

9. Piotrowski T, Milecki P, Skorska M, Fundowicz D. Total skin electron irradiation techniques: a review. Postepy Dermaol Alergol. 2013;30(1): $50-55$.
10. Olsen EA, Whittaker S, Kim YH, et al; International Society for Cutaneous Lymphomas; United States Cutaneous Lymphoma Consortium; Cutaneous Lymphoma Task Force of the European Organisation for Research and Treatment of Cancer. Clinical end points and response criteria in mycosis fungoides and sezary syndrome: a consensus statement of the International Society for Cutaneous Lymphomas, the United States Cutaneous Lymphoma Consortium, and the Cutaneous Lymphoma Task Force of the European Organisation for Research and Treatment of Cancer. J Clin Oncol. 2011;29(18):2598-2607.

11. Talpur R, Singh L, Daulat S, et al. Long-term outcomes of 1,236 patients with mycosis fungoides and Sézary syndrome from 1982 to 2009. Clin Cancer Res. 2012;18:5051-5060.

12. Scarisbrick JJ, Kim YH, Whittaker SJ, et al. Prognostic factors, prognostic indices and staging in mycosis fungoides and Sézary syndrome: where are we now? Br J Dermatol. 2014;170:1226-1236.

13. Smith BD, Wilson LD. Management of mycosis fungoides. Treatment: part 2. Oncology. 2003;17:1419-1428.

14. NCCN. Clinical Practice Guideline in Oncology: Non-Hodgkin's Lymphomas, Version 4; 2014. Available from: http://www.nccn.org/ professionals/physician_gls/f_guidelines.asp. Accessed April 9, 2015.

15. Navi D, Riaz N, Levin YS, Sullivan NC, Kim YH, Hoppe RT. The Stanford University experience with conventional-dose, total skin elecronbeam therapy in the treatment of generalized patch or plaque (T2) and tumor (T3) mycosis fungoides. Arch Dermatol. 2011;147(5):561-567.

16. Specht L, Dabaja B, Illidge T, Wilson LD, Hoppe RT; International Lymphoma Radiation Oncology Group. Modern radiation therapy for primary cutaneous lymphomas: field and dose guidelines from the international lymphoma radiation oncology group. Int J Radiat Oncol Biol Phys. 2015;92(1):32-39.

17. Harrison C, Young J, Navi D, et al. Revisiting low-dose total skin electron beam therapy in mycosis fungoides. Int $J$ Radiat Oncol Biol Phys. 2011;81(4):e651-e657.

18. Hoppe RT, Harrison C, Tavallaee M, et al. Low-dose total skin electron beam therapy as an effective modality to reduce disease burden in patients with mycosis fungoides: results of a pooled analysis from 3 phase-II clinical trials. J Am Acad Dermatol. 2015;72(2):286-292.

19. Kim YH, Liu HL, Mraz-Gernhard S, Varghese A, Hoppe RT. Long term outcome of 525 patients with mycosis fungoides and Sezary syndrome: clinical prognostic factors and risk for disease progression. Arch Dermatol. 2003;139:857-866.
OncoTargets and Therapy

\section{Publish your work in this journal}

OncoTargets and Therapy is an international, peer-reviewed, open access journal focusing on the pathological basis of all cancers, potential targets for therapy and treatment protocols employed to improve the management of cancer patients. The journal also focuses on the impact of management programs and new therapeutic agents and protocols on

\section{Dovepress}

patient perspectives such as quality of life, adherence and satisfaction. The manuscript management system is completely online and includes a very quick and fair peer-review system, which is all easy to use. Visit http://www.dovepress.com/testimonials.php to read real quotes from published authors. 